$\S=-1$

\title{
Comparison between Different Queue Management Techniques in Mobile Ad-Hoc Network: A Survey
}

\author{
Prabhat Kumar Sahu ${ }^{1 *}$, Susrita Mahapatro ${ }^{1}$, Pradeep Kumar Dash ${ }^{1}$ \\ ${ }^{I}$ Department of Computer Science and Engineering, \\ Institute of Technical Education \& Research, \\ Siksha 'O' Anusandhan University, Bhubaneswar, Odisha, India \\ *Corresponding author E-mail: prabhatsahu@soa.ac.in
}

\begin{abstract}
The world is running in a lightning speed with the rapid up gradation of technology. In fraction of second human civilization experiences a unique set of technologies and for this human sentiment started to demand better quality of service in almost all the areas which provides ease to it by minimizing human work efforts. Communication becomes an integral part of this rapidly changing technology. From the introduction of internet, the taste and preferences of virtual communication has been changed. A new technology called as Mobile Ad Hoc Network (MANet) is also getting its popularity due to its infrastructure less and mobility property. The rapid increase in demand for use of MANets tends it to further development to provide better quality of service. However Congestion in these networks creates a challenge to achieve desired Quality of Service. Different Queue Management techniques such as Passive and Active Queue management techniques are used to minimize the congestion in these networks which uses the concept of managing the buffers though algorithms. The Passive Queue Management technique Droptail, in the introduction stage, somehow manages to increase its efficiency. However in later stage, when data traffic increases, due to global synchronization problem many other algorithms including active queue management techniques such as RED and variants, REM have been developed to increase the efficiency by decreasing the congestion. The concepts even travel from congestion control to congestion avoidance. In this paper we have taken into account different passive and active queue management techniques and compared them based on our literature review to find out the best among them by considering different situations.
\end{abstract}

\section{Keywords: Droptail; AQM; MANET; PQM; QOS; RED; REM}

\section{Introduction}

A fraction of second is important in this rapidly changing era at least when it has a direct and significant impact on the human civilization. The modern technology gives us the opportunity to choose the best out of the best and to develop and maintain the standard in the best possible way. Communication is one of the field in which the world, in the recent era, witnesses rapid growth and development. Day by day the technologies modernize, amounting to better service quality along with minimizing the cost for service. Networking is a vital discipline of the communication system that integrates the end users communicating with each other. Networking, right now, is not limited to physical networking through wires or solid mediums, it has undergone rapid up gradation, enabling us to communicate wirelessly sometimes even without any fixed infrastructure.

Mobile Ad Hoc Networks (MANets) is one of such dynamic network topology that can be established at any place, at any point of time without any pre-infrastructure, in more detailed sense the network itself works as an infrastructure of communication having mobile nodes forming an energetic and temporary network without any base station.[1] The network consists of limited number of mobile nodes having a limited range of communication where by interacting with each other through wireless messages constitute by several data packets. Each and every node inside the commutation range is somehow responsible for the successful delivery of the data packets in the end nodes.[2] The nodes not only act as sender or receiver rather also act as routers and responsible for route discovery and route maintenance for other nodes.

The physical specifications of the nodes are different from the nodes connecting wired medium of communication. Basically the nodes are mobile devices or laptops, having limited resource for communication such as limited CPU cycles, storage area, battery capacity and even low bandwidth. [3]

These types of networks are basically useful and widely used for rapid interaction in case of emergency situations like search and rescue operations. They are also used in uncongenial territory where it is difficult to establish an Infrastructured network system.[4] Research is going to fully apply this technology in education, corporate sectors and even in multiplayer gaming systems.

The Mobile Ad Hoc Networks despite of several advantages could not be utilized in full phase due to lack in quality of service. This cost effective wireless and infrastructure less network never gives guarantee over the quality of service which sometimes resulted in interrupt in communication. Congestion in the network is one of the major causes for degradation in quality of service. The proposed paper discusses different congestion control mechanismand tries to find the best congestion control mechanism to improve the quality of service in MANets. [3] 


\section{Quality of Services in MANets:}

The word QoS signifies the efficiency of services provided by the mobile ad hoc network while transmitting data packets from the source node to the destination, sometimes directly or through intermediate nodes. It is evaluated using a number of parameters such as speed, time and drop.[5] A wide range of research is going on to maximize quality of service provide by such network, which possess a great challenge for the researcher due to the mobility of the nodes. It is a fact that mobility and lack of infrastructure make this network popular and even efficient in emergency situations; however QoS becomes a dilemma in utilising such network. The effective parameters which have significant impact on QoS are delay, delay variance (jitter), bandwidth, probability of packet loss, and power consumption. QoS is not only amounting to the successful delivery of data packets between end nodes, it also covers the security features, the speed of delivery to the desired node with optimum energy utilization.[6]

QoS, considering the definition providing by different authors, can also be defined as the degree of satisfaction of the end users depending on the performance of the network amounting to effective and successfully deliver of messages. Of course, both the above definitions focuses on one direction i.e. to improve the real time application of mobile ad hoc networks providing the best quality of services to the end users. [7] The problem here is there are no specific standard worldwide to measure the quality of service in MANets, however the goal of the researchers is to increase it to an extend where by the end users will never complain about or question the usability and applicability of this network at least in terms of quality of service.

\section{Congestion in MANets:}

Time is an important factor in real time communication. A delayed message from the sender to receiver carries no meaning rather sometimes create ambiguity and confusion. To be in time the sender has to encode and send the message within the prescribe time, receiver has to receive and decode the same within the allocated time but, here the main concern is to transmit the data packet from the sender to the receiver in limited time due to the complex structure of this type of networks arising out of the wireless medium and congestion is one of the major cause for delay in transmission amounting to lack in quality of service. To understand congestion we have to consider the complex structure of mobile ad hoc network in which every node are interconnected with each other either directly or indirectly through intermediate nodes in which every node having their own buffer to accept, send or hold the data packets. Again, each and every node is somehow related to transfer the data packets from one node to another and hence all the nodes are interdependent with each other through constituting a transmission path for every other node. Making it more complex every node is mobile in nature and hence continuously changes its position, which makes the transmission more difficult. The above mentioned complex structure is nothing but a catalyst for the congestion.

The term congestion refers to a complicated situation in which the flow of data from the source to destination either stops or slows down in a part of the subnet.[8] It occurs basically in two scenarios. Firstly, when the number of data packets more than the capacity of the network tries to utilize the same transmission path for packet forwarding. Due to heavy traffic the network slows down and hence a long delay occurs for delivery of the data packets.[9] Secondly, when the buffer present in an intermediate node overflows due to acceptance of more number of data packets a node can deliver or sent outside through the network to other nodes.[10] It is not disputed that some other factors like environmental change do have significant impact on data transmission amounting to congestion in the network, however they are situational based and can be overcome with the change in time and situation.
We have already discussed the complex structure and the physical challenges of this network which also includes limited buffer size. Mobility is the primary cause for which the size of the buffer cannot be extended as desired as it affects the size, shape and more over the power consumption of the mobile nodes. The limited size of the buffer, hence, became a cause for the congestion.

Congestion in mobile ad hoc network not only cause significant delay but also resulted in packet loss and bandwidth degradation. The congestion in MANets is different from wired network in a greater sense. In case of a wired network congestion normally occurs in a concentrated region of a single router or in a single routing path, but, as MANet uses a shared medium for communication hence, congestion targets and affects the entire network, sometimes affecting almost all the mobile nodes in a particular range. The following issues arose when congestion occurs and are not controlled effectively:

\subsection{Delayed Packet Delivery:}

The delay causes by the congestion can be categorized into two parts due to two different causes. The first one is because of the simple reason that the packet cannot be moved or transmitted through the intermediate channels due to the congestion and as a result the data transfer rate significantly drops delaying the delivery of the data packet. In simple term the actual delay caused by the congestion due to interrupt in data transfer.[11]Secondly, because of, the network tries to find the alternative paths to deliver the congested data packets into the destination node. Finding new paths and sending the data packets though the new route of course takes more time causing excess delay in transmission.

\subsection{High Overhead:}

Consider a scenario in which a communication takes place through a multipath channel and congestion occurred in two or more intermediate path simultaneously and to avoid the congestion the network tries to discover new routes. As we know the entire communication path is constituted by the contribution of every node in the range. [11] Hence the phenomenon of finding the new route affects all the nodes in the corresponding region and every node need to update their data stored in the form of the table. In this scenario the network suffers from higher overhead due to high resource allocation, bandwidth and energy consumption

\section{Control Mechanism for Congestion in MANets:}

The two scenarios contributing to the congestion has already been discussed above. Different authors prescribed different methods to control the congestion in mobile ad hoc networks. The control mechanism for MANets in a layman's term simply represents to overcome from the occurred congestion by utilizing a set of control algorithm. However scientifically and elaborately the term control mechanism defines a wide range of action and interaction including detection, prevention, overcome and even precaution for congestion in the network.[12] The idea behind the control mechanism is to ensure distributed bandwidth across multiple end to end nodes. It also ensures that no node in the entire system should send more number of data packets than the capacity of the network to transmit or the receiver can receive. [13] Some authors suggest the application of TCP (Transmission Control Protocol) to detect and overcome from congestion and some other trust upon the Queue Management Techniques.[14] TCP although used widely in the wired network successfully but contributes less to MANets because of the difficult application in the wireless and infrastructure-less network. Queue Management seems to be a better option because when we can't apply the old technology in a new medium of communication we can always hope and trust to 
develop and manage our own ways to overcome such problems for such new technology by contributing or upgrading to the current standing technology. In this paper we have observed and discussed a number of queue management techniques and try to find out the best among them to best fit in different situations.

\section{Queue Management Techniques:}

Avoiding or controlling Congestion through Queue Management techniques turns out to be a popular and cheaper method rather than designing and/or inserting a complete new algorithm for transmission. Communication using MANets is actually virtual in nature and a researcher working over this virtual network almost has no chance except working over the algorithms. Algorithms in such networks are the lifelines, and highly responsible for the intercommunication.[15] We consider the network as much as realistic to work over because it is the human nature that indeed them to always work over a tangible object not on an imaginary based. Queue Management in the realistic term is nothing but a way to work on the nomenclature of the network, simply working over the algorithms responsible for data transmission to make such transmission hassle free without any congestion.[16] The objective is simple. We need to either eliminate congestion or prevent congestion by ordering the data packets correctly or in an alternative way by limiting the data packets through one exclusive indication by prioritizing the data packets. The more priority the data packets has the more chance of its delivery and the least priority data packets, in the worst case, considered to be dropped.[17] The mechanism for the queue management overly worked as such and basically classified into two:

\section{A. Passive Queue Management \\ B. Active Queue Management}

\subsection{Passive Queue Management:}

The classifications of the Queue Management techniques are based on the mechanism of dropping packets in case of severe congestion. Active and Passive Queue Management techniques are different in two senses. In both the algorithm the strategy for dropping data packets are different and the indication to the source node about the drop of the data is carried out in a different way. The buffer present in the intermediate nodes and even in the receiver has a limited size or length and hence can accept data packets to certain extend.[18] This length size in the Queue is known as maximum buffer length. A new data packet is allowed to enter into the buffer only when the length of the data packets, combining the length of the entering data packets with the length of the data packets present in the buffer, is less than the maximum buffer length. In other words transmission from one to another node is allowed when the number of the data packets carried in the second node is less than the capacity of the second node that can carry up to a fixed number of data packets. When the buffer is full or in its highest capacity, the incoming data packets are dropped till a point when some portion of the length of the queue is free. The source gets the idea about the congestion only when some data packets are dropped because the technique never uses an explicit mechanism to detect the congestion or calculate it before at least drop of a single data packet.[19] In PQM, there is no way that the source can predict about the congestion in the network before data drop is started, which is the major disadvantage of this type of network. The advantage is such techniques are easy to implement and maintain over a time and minimizes overhead in the network in case of less data traffic. Droptail, DEC Bit, SFQ (Stochastic Fairness Queuing) are the techniques implemented passive queue management technique.

\subsubsection{Droptail:}

Droptail, a passive queue management technique, follows the mechanism of First in First out (FIFO) policy to enqueue and dequeue data packets to/from the buffer. This theory was proposed by RadeStanojevic et al while working on congestion control mechanism in MANets.[20] When data from the previous node arrives the algorithm makes it enter into the tail of the queue and after processing i.e finding the next suitable node for transmission, in case of the node acting as an intermediate node, send it through the head of queue to the next node. This theory holds good till the length of the queue is not full and when it is full, this algorithm prohibits the incoming data packet and drops it to control the network from congestion. The drop of the data packets happens at the tail side of the queue and hence the name is so called as "Droptail". The sender in the meantime detects the drop of the packet in the transmission path and shrinks its sending window to slow down the data transfer. The implementation of the algorithm is so simple to utilize and efficient enough where the data transfer is limited over a period of time. However this algorithm is not suitable when the computation overhead is very high in case of large volume of data transfer among the nodes. Continues dropping of the data packets leads to high packets loss, decrease in throughput as well as high end to end delay.

This Algorithm is the backbone for other algorithms. It is easy to implement but if the computational overhead is more then it cannot be implemented. When continuous dropping of packets take place due full of buffer, it suffers from high packet loss and decreased throughput. The main disadvantage of Droptail is it suffers global synchronization problem. The algorithm although in some cases suitable for congestion control but completely fails in case of congestion avoidance.

\subsubsection{DEC bit:}

DEC bit uses one extra binary bit in the packet header to get the information about congestion in the network. In this method we are calculating average queue length according to the last busy and ideal time period. Here Average Queue length is the length of the data packets including the packets inside the buffer with addition to the packets incoming to the buffer. The route buffer size is fixed and signifies the maximum length of the buffer which can contain the data packets up to certain limit. When Avg_queue length > route _buffer size, then the binary bit is set to 1 to signify congestion is going to occur and inform the source regarding the status of the buffer in the transmission channel.[21] The source uses window flow control mechanism, each time it updates the window of data packets. The size of the window in the source node can be increased or decreased exponentially or linearly depending on the remaining buffer size at the intermediate node where congestion is going to be happened. A DEC bit 1 made the source to shrink its window and slow down the data transfer rate. Exceptionally when the binary bit is set to 1 and the sender has to send little more packets, the congestion window is divided to half continuing the data transfer with the DEC but set to 1 .

\subsubsection{SFQ:}

SFQ or Stochastic fairness queuing is an innovative passive queue management technique, which implements the fair queuing algorithms. The fairness is in terms of packet delivery.[22] The algorithm is so called because it depends on hypothetic allocation of queue in each section which means in partial it does not allocate a queue rather it implements an innovative algorithm which is used to divide the traffic over a number of queues which are limited in number. This algorithm works over the concept of hashing. The concept includes the less accuracy than other queuing techniques however it requires less calculation resulting to fewer loads while being perfectly fair.

\subsection{Active Queue Management:}

We look for and get solutions when we realise the problem. The passive queue management technique although holds good for less traffic area but creates a gigantic problem. When the traffic 
increases it leads to congestion and the nodes required dropping packets which resulted in high packet loss and minimize the efficiency of the whole network. As we earlier said solution came after problem the solution here is to drop excess packets before the queue becomes full and to slow down or even stop when the congestion is going to occur. [23]

Active Queue Management uses small size steady state queue to prevent itself from the issue occurred in Droptail. The primary goal of utilizing active queue management is to reduce the range of drop packets, minimize end-to-end delay, minimize the waste of obtained bandwidth and increase the link utilization. This queue management utilizes the concept of getting knowledge of the congestion before it going to be happened in the network and take necessary action to avoid the congestion. The algorithms used for AQM are complex and difficult to implement, however are efficient enough in case of heavy traffic and high computational overhead.[24] The algorithm believes in congestion avoidance rather than congestion control. The source node in this case plays a great role to control the flow of the data packets according to the predicted congestion. The algorithm uses permutation and combinations along with probability to compute the congestion which is going to be happened in the network.

Different Active Queue Management schemes have been proposed in the recent era and some of them are RED, GRED, NLRED, MRED, TRED, REM.

\subsubsection{RED (Random Early Detection):}

RED or Random Early Detection is an active queue management technique which implements the technique of congestion avoidance depending on the length of the queue.[25] This algorithm was proposed by Floyd and Jacobson to solve the problems which occurred in passive queue management techniques.[26] The mechanism monitors the size of the queue and randomly marks or drops the data packet depending on the statistical probability of congestion in the network. This mechanism is based on prevention rather than solution that it believes in slowing down the flow of data rather than dropping it, when possible.The statistical probability is being calculated according to the size of the buffer and the data packet is to be routed through the node. The probability function in case of the RED is linear in nature and increase with the measure of congestion function. If the size of the buffer is empty it accepts all the data packets. As the packets occupies the buffer the probability of dropping the packets also increases and subsequently reach to one which indicates to drop the packets or to mark the packets that the transmitter can slow down the process of sending data. It helps to avoid TCP global synchronization.

\subsubsection{GRED (GENTLE RED):}

The Gentle RED is another variant of RED queue management technique which believes in the mechanism of gently increase the probability to 1 depending upon the average queue length and the packet dropping probability.[27] Here the packet dropping probability increases linearly to 1 only when the average queue length exceeds twice of the maximum length of the queue. The concept although seems easy but the implementation in real time environment is difficult and not feasible in every case. Linear function can't be used in every environment due to the increase in complexity of implementation and energy consumption due to high mathematical overhead.

\subsubsection{ARED (ADAPTIVE RED):}

The idea behind developing Adaptive RED algorithm is to minimize the delay and to achieve high throughput by modifying the average queue size and by formulating a set of formulas for the same, where the average queue size is being calculated by $(\mathrm{qmin}+0.4 *(\mathrm{qmax}-\mathrm{qmin}), \mathrm{qmin}+0.6 *(\mathrm{qmax}-\mathrm{qmin})) \cdot[28]$ To maintain the stability of the average queue length the mechanism of adaptive increase andmultiplicative decrease policy is used. The maximum probability Pmax can be calculated as follow:

Where Pmax $<0.5$,

Then $\mathrm{Pmax}=\mathrm{Pmax}+\alpha$, qavg $>$ qtarget

When Pmax $>=0.01$,

Then $P \max =P \max * \beta$, qavg $<$ qtarget

\subsubsection{NLRED (NONLINEAR RED):}

As we discuss earlier one of the major problem in MANET is to optimize the performance by minimizing the congestion by packet drop. However one truth prevails, packet drop cannot be stopped even in the ideal condition, due to the frequent change of the atmospheric and humanistic condition. The traditional RED algorithm uses linear function to drop the data packets. The Nonlinear RED or NLRED used a different concept of dropping data packets using nonlinear function when avg_1 is in between minimum and maximum.[29]

\subsubsection{TRED (THREESECTION RED):}

RED algorithm although blessing for the modern adhoc network system but distinguished in terms of certain areas like high packet drop ratio, degradation of throughput and high probability of link failure. However all of the above are dependable variable and basically depends on the size of the queue. RED, the advance queuing algorithm uses the mechanism in which the size of the queue varies with the level of congestion and leads to one of the major drawback of the algorithm. This leads to an unpredictable queuing delay in RED. The TRED or Three sections Random Early Detection has been developed to overcome from this problem. This algorithm uses the concept of dividing the packet dropping probabilities into three equal sections and named it as light, medium and high load.[30] When it detects light load it allows the resources to use the queue rather notify high load network to use its queue to minimize high loads in other networks. The medium load is considered as the ideal load condition and in this condition the algorithm has to do nothing except trying to maintain the same condition. In case of high load condition it drops packet and tries to decrease the congestion and delay.

\subsubsection{MRED (MODIFIED RED):}

Modified RED is the advance version of TRED which uses an ECN (Explicit Congestion Notification) to make the algorithm more reliable and optimum. As we discussed above the TRED categorize the network load into three subsections like low, medium and high load sections. This algorithm adds the concept of ECN into all these three sections. In case of low load section it requests the source to send high number of data packets to make high path utilization which ultimately minimizes the delay. In case of medium load it acts similar as TRED.[31]However in case of high load section this algorithm acts differently i.e either it sends an ECN notification to the source node to decrease the sending data rate or in the worst condition it drops the data packets. This algorithm believes in notifying the sender rather than dropping the data packets. The major disadvantage of this algorithm is the complicacy of using ECN algorithm for which the setup of the nodes must be capable of using ECN mechanism. Again to make each and every node ECN capable is not feasible in terms of costbenefit ratio. However if we compare it with other active queue management techniques and even with any passive queue 
management technique, MRED is still a better option for queue management due to its advantages.

\subsubsection{BLUE ALGORTHIM:}

The mechanism of RED algorithm is especially based upon the calculation of instantaneous average queue length but the BLUE algorithm especially based upon the mechanism of packet loss and link utilization. In case of this algorithm when the buffer is over flowed the marking probability is increased which leads to increase in packet drop ratio. The major disadvantage of this algorithm is that the flow is tented forever, when it is once marked which leads to packet drop even when flow retrains itself later on.[31]

\subsubsection{REM (Random Exponential Marking):}

Random Exponential Marking or REM works over the same concept as RED. Both RED and REM focuses on congestion avoidance rather than congestion control. The difference is in terms of measuring the congestion and marking the data packets using marking probability function. This algorithm proves itself as the best in terms of bandwidth utilization and delay. In case of heavy traffic and high overhead, REM performs better than other algorithm as it shows high bandwidth utilization with minimum end to end delay and packet loss.[32] The two key features contributed to the efficiency of the network are:

\section{a. Match Rate Clear Buffer \\ b. Sum Prices}

\subsubsection{Match Rate Clear Buffer:}

Match Rate Clear Buffer uses the concept of matching the user rate with the network capacity regardless of the number of users. It uses the concept of stabilizing both the input rate and the queue regardless of uses count. The variable used for measuring the congestion in REM is known as "Price". Price is used to determine probability of congestion or the marking probability. An algorithmic function periodically or asynchronously updates the value of the price with each successful or failed transaction by considering the rate mismatch and queue mismatch. The rate mismatch is calculated from the difference in the rate of input and capacity of link. Accordingly the queue mismatch is calculated by the difference in length of the queue and targeted length. The price increments with the detection of positive weighted sum of the "mismatches", and vice versa.[32] When Price increases it marks the data packets and sends the signal to the source to decrease the data sending rate and vice versa in case of decrease in price. This helps the algorithm to attempt high bandwidth utilization and decreases delay as well as packet loss.

\subsubsection{SUM PRICE:-}

The idea behind the sum price is to calculate the sum of the link prices in the travelled path to measure the congestion in the path. It uses end-to-end marking probability and informs it to the source to control the data flow which also controls the data flow when congestion occurs. Congestion in this case can be prevented by explicitly notifying the source through marking the data packets.[32] Source may increase and decrease the flow of the data packets considering the message received through the marked data packets.

\section{A brief comparison table}

Table 1: Comparison amongst different active and passive Queue management techniques.

\begin{tabular}{|c|c|c|}
\hline CLASSIFICATION & ALGORITHIM & $\begin{array}{l}\text { HIGHLIGHTED } \\
\text { DESCRIPTIONS }\end{array}$ \\
\hline \multirow{3}{*}{$\begin{array}{l}\text { PASSIVE QUEUE } \\
\text { MANAGEMENT } \\
\text { TECHNIQUE }\end{array}$} & DROPTAIL & $\begin{array}{l}\text { - The Simplest Algorithm } \\
\text { to execute, maintain and } \\
\text { modify. } \\
\text { - Packets are dropped } \\
\text { only in case of } \\
\text { congestion } \\
\text { - State Information is not } \\
\text { needed and best for low } \\
\text { traffic use. } \\
\text { - Suffers from } \\
\text { Synchronization problem } \\
\text { - No Congestion } \\
\text { Avoidance mechanism. }\end{array}$ \\
\hline & DEC BIT & $\begin{array}{l}\text { - Uses one extra bit in } \\
\text { packet header to notify } \\
\text { congestion. } \\
\text { - Feedback through } \\
\text { traffic marking packets. } \\
\text { - Better control for } \\
\text { congestion with increase } \\
\text { in fairness. } \\
\text { - Low } \\
\text { utilization. } \\
\text { - No bandwidth } \\
\text { Avoidance mechanism. }\end{array}$ \\
\hline & SFQ & $\begin{array}{l}\text { - Innovative technique } \\
\text { using fairing queue } \\
\text { Algorithm. } \\
\text { - Hypothetic Allocation } \\
\text { of Queue Space } \\
\text { - Look up cost is } \\
\text { significantly reduced. } \\
\text { - Complexity increases } \\
\text { due to increase in } \\
\text { number of queues. } \\
\text { - Not so fair in heavy } \\
\text { traffic conditions } \\
\text { - No Congestion } \\
\text { Avoidance mechanism. }\end{array}$ \\
\hline \multirow[t]{2}{*}{$\begin{array}{l}\text { ACTIVE QUEUE } \\
\text { MANAGEMENT } \\
\text { TECHNIQUE }\end{array}$} & RED & $\begin{array}{l}\text { - Implements both } \\
\text { congestion avoidance } \\
\text { and control mechanism. } \\
\text { - Probability function is } \\
\text { used to detect congestion } \\
\text { and accordingly packets } \\
\text { are marked to prevent } \\
\text { congestion. } \\
\text { - Avoids TCP global } \\
\text { synchronization } \\
\text { problem. } \\
\text { - Unbiased in case of } \\
\text { bursty traffic even in } \\
\text { high overload. } \\
\text { - Complex in } \\
\text { implementation and } \\
\text { sensitive to parameters } \\
\text { received }\end{array}$ \\
\hline & GRED & $\begin{array}{l}\text { - Modified variant of } \\
\text { RED algorithm. }\end{array}$ \\
\hline
\end{tabular}




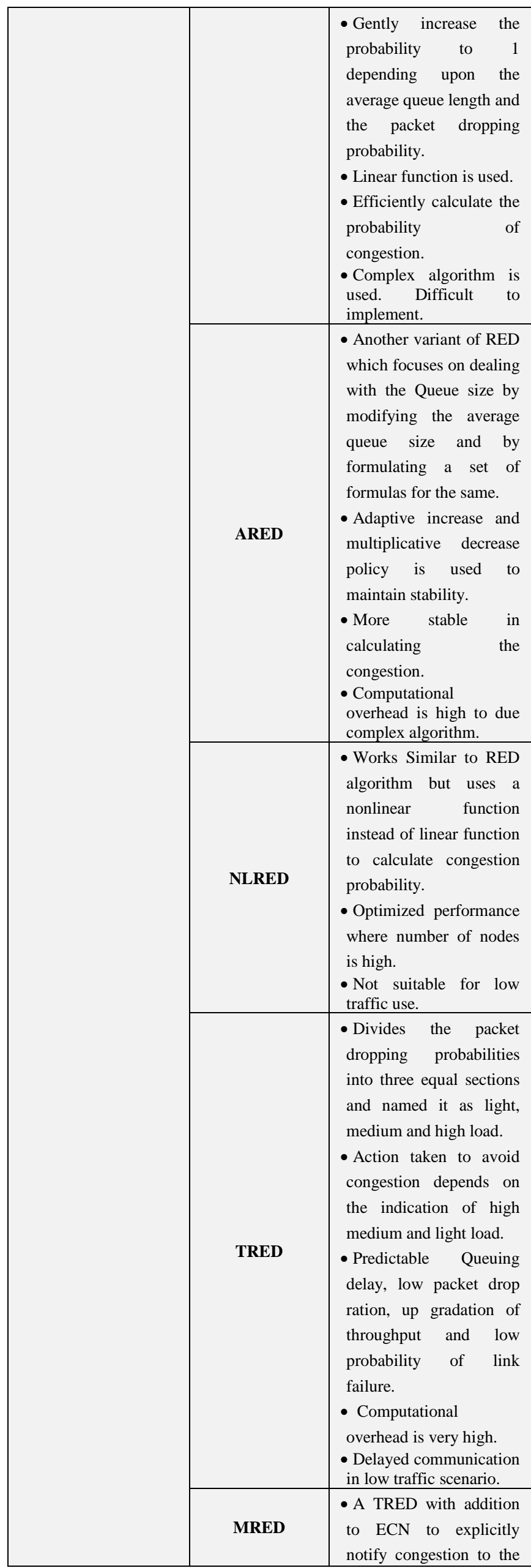

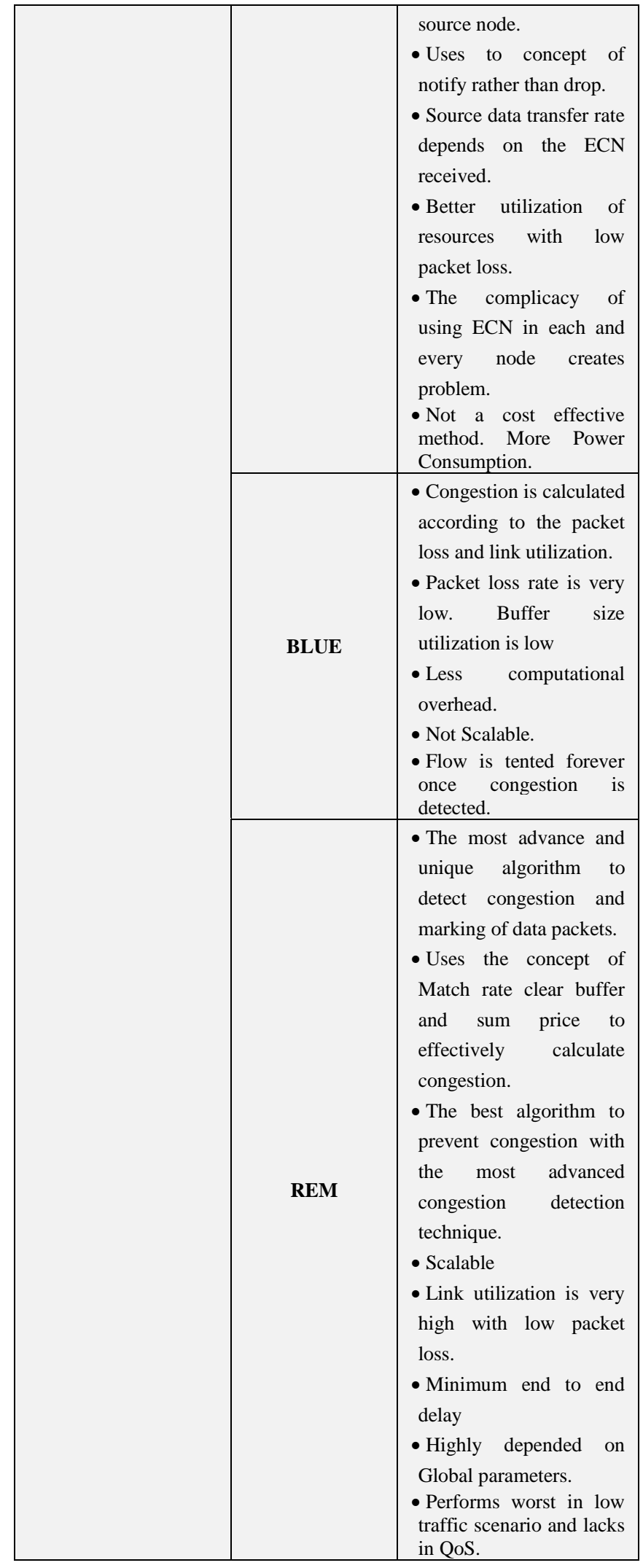

\section{Conclusion:}

Nothing is permanent in this world and Nothing is hundred percent efficient. Likewise our study suggested that there is no algorithm which can be termed as the winnerby considering different situations. In this paper we take in to account both passive and active queue management techniques to study their efficiency. Some are efficient in low traffic conditions and some works well over high traffic conditions. Some of them cease the work when computational overhead becomes very high. The study 
shows that in case of low traffic and less overhead passive queue management technique, Droptail, performs the best but performs the worst in case of heavy traffic. Droptail is also found out to be the simplest and easiest algorithm to implement. This algorithm may be used, in case of MANets, for the purpose of education and gaming where the numbers of users are predetermined with the determination of the fixed data transfer rate. Basically in case of educational and gaming use data transfer is uniform to different nodes and can be determined prior to the use and hence Droptail is the best choice here. The study further suggests that RED and its variants, considering as a whole, happen to be the best algorithm when the traffic in the network is heavy. It is because of its ability to maintain stable queue size which helps to lower the delay and tends to achieve high throughput and link utilization. Amongst RED and its variants, NLRED (Non- Linear Random Early Detection) wins the race when we consider the performance with respect to queue size and average throughput. RED and NLRED can be used for communication in case of emergency situation which demands heavy traffic flow. REM, although the newest algorithm which implements unique techniques, sometimes lacks in quality of service because it highly depends of the global parameters. Further studies may require increasing the efficiency of REM and its proper utilization. Considering the practicality of use of these algorithms depending on the complexity Droptail as well as RED can be used, of course, the choice is highly based on the data traffic, cost and situation for implementation.

\section{Future Scope:}

An Author should consider, while comparing different queue management techniques, the dynamics and the feasibility of application of such techniques. The dynamics of the queue management techniques were discussed briefly in this paper. However a vast scope is there to analyse and compare the techniques in terms of the feasibility which includes the cost of application, cost of implementation and also the practicability of such queue management techniques. Secondly, in this dynamic and fast traversing world may be while drafting this research paper, in some corner of the earth, a new queue management technique is being evolved and maybe serve the purpose in a better way.The authors who will study the same in future can include those new techniques andmay conclude with a different dynamic observation. We hope and trust further studies in this direction will help us to minimize congestion in the network to an extent where communication will be hassle free with minimum cost for service.

\section{References}

[1] M. Conti, and S. Giordano, "Mobile Ad Hoc Networking: Milestones, Challenges, and New Research Directions," in IEEE Communications Magazine, Vol.52, Issue: 1, January 2014. pp $85-96$

[2] P. Panchal, and M. Shroff, "Mobile Ad Hoc Networks," in International Journal of Students' Research in Technology \& Management, Vol. 2(05), August-September, 2014,pp 183-187

[3] N. S. Kavitha, P. Malathi, G. Poovila, and J.M. Philip, "Survey on Congestion Control in Mobile Ad Hoc Networks," in International Journal of Advanced Research Trends in Engineering and Technology (IJARTET), Vol. 3, Special Issue 2, March 2016

[4] S. G. Datey, and T. Ansari., "Mobile Ad-Hoc Networks Its Advantages and Challenges," in International Journal of Electrical and Electronics Research, Vol. 3, Issue 2, 2015 , pp: 491-496.

[5] S. Rakas,V. and Timcenko, "Quality of Service and Security Issues in MANET Environment," in 22nd Telecommunications forum TELFOR, Serbia, Belgrade, November, 2014 pp:25-27.

[6] M. Khan, and I. Das., "A Study on Quality of Service Routing Protocols in Mobile Ad Hoc Networks," in International Conference on Computing and Communication Technologies for Smart Nation, 2017.
[7] P.K Sahu, B.K Pattanayak, "Quality of Service based Multicasting Routing Protocols for MANets: A Survey," in Internation Journal on Communications Antenna and Propagation, Vol. 7, Issue: 5 Oct 2017pp:423-438

[8] S. Leemaroselin, "A review on congestion control algorithm in MANets,"inInternational Journal of Computer Science \& Engineering Technology, Vol. 6, No.04, Apr 2015.

[9] G. Maheshwari,M. Gour, and U. Chourasia, "A Survey on Congestion Control in MANET," International Journal of Computer Science and Information Technologies, Vol. 5 (2), 2014.

[10] D. Gupta, and M. Shrama, "A Survey on Congestion Control on Mobile Ad-Hoc Networks," International Journal of Engineering Research \& Technology, Vol. 3 Issue 6, June 2014

[11] S. Sheeja, and R. Pujeri "Effective Congestion Avoidance Scheme for Mobile Ad Hoc Networks," in I. J. Computer Network and Information Security, Vol.1, 2013 pp: 33-40.

[12] P. Dhibya,and S. Meenakhi, "A Review of Congestion Control Techniques in Mobile Ad-hoc Network," in International Journal of Computer Sciences and Engineering, Vol.3,Issue-9, 2015

[13] S. Tiwari, A. Jain , and Y. Rana, "A Survey on Congestion Control Mechanism in Mobile Ad Hoc Networks," in International Journal of Scientific \& Engineering Research, Vol. 4, Issue 10, October-2013

[14] N. Sharma,A. Gupta, M. Rajput, and V. Yadav, "Congestion Control Technique in MANet: A survey," in Second International Conference on Computational Intelligence \& Communication Technology, 2016.

[15] P.K Sahu, B. K. Pattanayak, "Comparative Performance Analysis of ZRP Routing Protocol for Multicasting in MANets using Droptail and RED queue Management," in Far East Journal of Electronics and Communications, Vol: 17, Issue:2 , April 2017, pp: 295-310

[16] W. Wei, H. Song, H. Wang, and X. Fan, "Research and Simulation of Queue Management Algorithms in Ad Hoc Networks UnderDDoS Attack," in IEEE Access Vol. 5, March 2017, pp 27810 - 27817

[17] S. Kushwash, and U. Dwivedi,"Analysis of Dynamic Buffer Management Approaches in Mobile Ad hoc network," in International Journal of Engineering and Computer Science Vol.6 No-9 Sep 2017

[18] S. Kochher, M. Sanghal, R. Kochher, and G. Singh, "A Review on Active and Passive Queuing Techniques," in International Journal for Science and Emerging vol. 15(1), 2014 pp:16- 22.

[19] P. Dhivya, and S. Meenakshi, "A Review of Congestion Control Techniques in Mobile Ad-hoc Network," in International Journal of Computer Sciences and Engineering, Vol-3, Issue-9 Sep 2015

[20] R. S. Rade, R. N. Shorten, and C. M. Kellett "Adaptive Tunning of Drop tail buffers for Reducing Queuing Delays," in IEEE Communication, Vol.10, No.7, July 2006, pp 570-572.

[21] T. Ziegler, and H.D. Clausen, "Congestion avoidance with BUC (buffer utilization control) gateways and RFCN (reverse feedback congestion notification)," inIEEE Xplore, Aug 2002

[22] G. Carlucc, L. D. Cicco, and S. Mascolo, "Controlling Queuing Delays for Real-Time Communication: The Interplay of E2E and AQM Algorithms" 2015

[23] https://pdfs.semanticscholar.org/5b0c/4263b70633979257b1a90 08e9dd98e490b45.pd

[24] A. K. Sharma, and A. K. Behra, "A Survey on Active Queue Management Techniques," in International Journal Of Engineering And Computer Science, Volume 5, Issue 11, Nov. 2016, pp. 18993-18997.

[25] S. K. Mohapatra , S. K. Bisoy, and P. K. Dash, "Stability Analysis of Active Queue Management Techniques," in International Conference on Man and Machine Interfacing, 2015.

[26] R. Agrawal, and P. Sharma, "Study of Various Queue Management and Random Early Detection Technique in Mobile Ad Hoc Network," in International Journal of Recent Development in Engineering and Technology, Volume 5, Issue 5 , May 2016.

[27] S. Floyd, and V. Jacobson, "Random early detection gateways for congestion avoidance," in IEEE Transactions on Networking, Vol.1, No.4, Aug 1993, pp. 394-413

[28] D. Zhang, Z. Ma, D. Zhao, H. Song, and S. Liu "Novel Adaptive Queue Intelligent Management Algorithm," in IEEE Xplore, Oct $\mathbf{2 0 1 5}$ 
[29] E. Natsheh, B.J. Adznan, S. Khatun, and S. Subramaniam, "Fuzzy Active Queue Management for Congestion Control in Wireless Ad-Hoc," in The International Arab Journal of Information Technology, Vol. 4, No. 1, January 2007

[30] C. Feng, L. Huang , C. Xu , and Y. Chang , "Congestion Control Scheme Performance Based on Nonlinear RED," in IEEE System Journal, Vol. 11, NO. 4, DECEMBER 2017.

[31] K. Vaghela, "Improved Congestion Control using Modified RED Algorithm over MANet," in International Journal of Engineering Development and Research, Volume 4, Issue 2, 2016.

[32] D. Saha, W. Feng, D. D. Kandlur, and K.G. Shin, "A New Class of Active Queue Management Algorithms, BLUE," in Technical Report CSETR- 387-99, University of Michigan, April 1999, pp: 1-27.

[33] L. Chros, and A. Chydzinsk, "On the deterministic approach to active queue management," in Springer Telecommunication Systems, Vol:3, Issue:1, Sep 2016, pp: 27-44 